\title{
SH3-domain mutations selectively disrupt Csk homodimerization or PTPN22 binding
}

Ben F. Brian IV ${ }^{1 \dagger}$, Frances V. Sjaastad ${ }^{2 \ddagger}$, Tanya S. Freedman ${ }^{2,3,4,5^{*}}$

${ }^{1}$ Graduate Program in Molecular Pharmacology and Therapeutics, University of Minnesota; Minneapolis, MN 55455, United States.

2Department of Pharmacology, University of Minnesota; Minneapolis, MN 55455, United States.

${ }^{3}$ Center for Immunology, University of Minnesota; Minneapolis, MN 55455, United States.

${ }^{4}$ Masonic Cancer Center, University of Minnesota; Minneapolis, MN 55455, United States.

${ }^{5}$ Center for Autoimmune Diseases Research, University of Minnesota; Minneapolis, MN 55455, United States.

${ }^{+}$Current Address: Division of Immunology \& Pathogenesis, Department of Molecular and Cell Biology, University of California, Berkeley; Berkeley, CA 94170, United States.

${ }_{\ddagger}^{\ddagger}$ Current Address: Department of Cardiac Rhythm Management, Medtronic; Mounds View, MN 55112, United States.

*Corresponding author e-mail: tfreedma@umn.edu (TSF)

Short Title (50 characters): Csk dimerization and PTPN22 binding 


\section{Abstract}

The kinase Csk is the primary negative regulator of the Src-family kinases (SFKs, i.e., Lck, Fyn, Lyn, Hck, Fgr, Blk, Src, Yes), phosphorylating a tyrosine on the SFK C-terminal tail that nucleates an autoinhibitory complex. Csk also binds phosphatases, including PTPN12 (PTP-PEST) and immune-cell PTPN22 (Pep/LYP), which dephosphorylate the SFK activation loop to promote autoinhibition. High local concentrations of Csk are required to promote its negative-regulatory function, and Csk-binding proteins (e.g., CBP/PAG1) oligomerize within membrane microdomains. Purified Csk also homodimerizes in solution through an interface that overlaps the phosphatase binding site. Here we demonstrate that Csk can homodimerize in Jurkat T cells, in competition with PTPN22 binding. We designed SH3-domain mutations in Csk that selectively impair homodimerization (H21I) or PTPN22 binding (K43D) and verified their kinase activity in solution. Csk W47A, an SH3-domain mutant commonly used to block PTPN22 binding, also impairs homodimerization. Csk H21I and K43D will be useful tools for dissecting the protein-specific drivers of autoimmunity mediated by the human polymorphism PTPN22 R620W, which impairs interaction with both Csk and with the E3 ubiquitin ligase TRAF3. Future investigations of Csk homodimer activity and phosphatase interactions may reveal new facets of SFK regulation in hematopoietic and non-hematopoietic cells.

\section{Introduction}

The protein tyrosine kinase Csk negatively regulates Src-family kinases (SFKs) by phosphorylating the Cterminal inhibitory tail to promote assembly of an autoinhibited state ${ }^{1-3}$. Unlike the SFKs, Csk lacks membraneanchoring lipidation sites, and its activity is not regulated by phosphorylation of a kinase-domain activation loop 1 . Instead, Csk is recruited to membrane-anchored SFKs and activated allosterically via its SH2 domain, which binds SFK-phosphorylated motifs in CBP/PAG1 (Csk binding protein/phosphoprotein associated with glycosphingolipid-enriched microdomains), paxillin, Fak, the Dok family, and other tyrosine-phosphorylated proteins ${ }^{1,4-7}$. Csk has low intrinsic activity toward peptide substrates and requires a stable interaction through an extensive binding interface for optimal substrate phosphorylation ${ }^{8}$. Csk localization and local concentration are thus key features governing its activity and substrate specificity.

Like the $\mathrm{SH} 2$ domain, the $\mathrm{SH} 3$ domain of Csk is required for kinase activity and for mediating protein-protein interactions $^{9-11}$. Primary ligands are PXXP-containing tyrosine phosphatases, including broadly expressed PTPN12 (PTP-PEST) $)^{12}$ and hematopoietic-cell PTPN22 (LYP in humans, Pep in mice) ${ }^{10}$. PTPN12 binds the $\mathrm{SH} 3$ domain of Csk via a canonical polyproline $(\mathrm{PXXP})$ interaction $\left(\mathrm{K}_{d} \sim 8 \mu \mathrm{M}\right)$, whereas the SH3-binding peptide of PTPN22 has a more extensive footprint, wrapping around the SH3 domain to bind with 10-fold higher affinity $\left(\mathrm{K}_{d} \sim 0.8 \mu \mathrm{M}\right)^{12,13}$. Along with Csk, PTPN12 and PTPN22 are critical modulators of homeostatic signaling and negative feedback to restrain SFK activity and reverse the phosphorylation of SFK substrates ${ }^{10,12,14}$.

In T lymphocytes the dominant SFK, Lck, phosphorylates and activates the downstream kinase Zap70 and ITAMs (immunoreceptor tyrosine-based activation motifs) within the T-cell antigen receptor (TCR) complex ${ }^{15-17}$. The activity of Lck is tuned to provide homeostatic function and inducible TCR signaling. Csk and PTPN22 are both required to maintain this balance. PTPN22 dephosphorylates the activation loop of Lck, the ITAMs of the CD3 3 TCR subunit, and the activation loop of Zap7018-20, adding a second level of TCR signal inhibition to the direct activity of Csk on Lck. Dysregulated Lck/TCR/Zap70 signaling, either too strong or too weak, can lead to autoimmune disease ${ }^{21}$, and Csk or PTPN22 dysregulation can promote autoimmunity and cancer ${ }^{22-25}$. Impairment of PTPN22 binding to the SH3 domain of Csk, as with an arginine-to-tryptophan substitution at position $620(\mathrm{R} 620 \mathrm{~W})^{26,27}$, is linked to autoimmune disease in humans and mice ${ }^{28-31}$.

The role of the wild-type (WT) PTPN22/Csk complex in T-cell and other cell signaling is not yet clear ${ }^{32}$. In some reports, binding to Csk appears to sequester and functionally suppress WT PTPN22, and the R620W variant is a gain-of-function allele ${ }^{32-36}$. Others have found the opposite, that the complex with Csk promotes PTPN22 function by colocalizing it with substrates ${ }^{10,37-39}$. In this latter model, PTPN22 R620W is a loss-of-function allele. The contributions of cell-type and species differences to these disparate reports is unclear. Further complicating these analyses, R620W also blocks PTPN22 binding to the E3 ubiquitin ligase TRAF3. In addition to promoting NF-KB signaling ${ }^{40,41}$, TRAF3 normally has an adaptor-like function binding and inhibiting Csk and PTPN22, potentially as a ternary complex ${ }^{42}$. 
Csk homodimer formation could limit the abundance of WT PTPN22/Csk complexes and contribute to PTPN22 R620W autoimmune disease. Purified Csk protein homodimerizes in solution with low-to-moderate affinity ( $\mathrm{K}_{d}$ $\sim 10-20 \mu \mathrm{M}^{43}$ ). This interface, mediated by symmetrical SH3-SH3 interactions that bridge two Csk molecules ${ }^{44,45}$, overlaps completely with the PXXP docking site for PTPN12 ${ }^{12,44}$ and overlaps substantially (but not completely) with the extended binding site for PTPN22 ${ }^{13,43}$. In cells, phosphorylated CBP/PAG1 can oligomerize, binding multiple molecules of Csk in supercomplexes localized to membrane microdomains or rafts $^{45}$. At these high local concentrations Csk homodimer interactions could compete with phosphatases for access to SH3-domain binding sites. However, Csk homodimerization and competition with PTPN22 in cells has not previously been investigated.

Here we report that Csk self-association in Jurkat T cells can compete with PTPN22 for binding to the Csk SH3 domain. Using structural analysis, we identified a histidine-to-isoleucine substitution (H21) that blocked coimmunoprecipitation of Myc- and HA-epitope-tagged Csk. This substitution increased co-immunoprecipitation of endogenous PTPN22. A lysine-to-aspartate substitution (K43D) in the SH3 domain of Csk blocked PTPN22 binding without disrupting Csk self-association. Neither mutation impaired the activity of purified Csk in solution. The tryptophan-to-alanine substitution (W47A) used previously to block PXXP-Csk interactions ${ }^{46-50}$ disrupted both PTPN22 binding and Csk homodimer formation. Together, our studies demonstrate that Csk can homodimerize in cells and restrict the formation of Csk/PTPN22 complexes.

\section{Results}

Multiple molecules of epitope-tagged Csk co-immunoprecipitate from Jurkat T cells

We investigated Csk self-association in Jurkat T cells by co-transfecting HA-tagged Csk (Csk ${ }^{\mathrm{HA}}$ ) and Myctagged Csk (Csk ${ }^{\mathrm{Myc}}$ ) and subjecting cell lysates to anti-Myc immunoprecipitation. Csk ${ }^{\mathrm{HA}}$ was detected at levels above background in $\mathrm{Csk}^{\mathrm{Myc}}$ immunoprecipitates, potentially reflecting co-immunoprecipitation of $\mathrm{Csk}^{\mathrm{HA}} / \mathrm{Csk}^{\mathrm{Myc}}$ dimers (Fig 1a). Endogenous PTPN22 ${ }^{19,26,51}$ also co-immunoprecipitated with Csk ${ }^{\text {Myc }}$ but was not detected in vector-only or Csk ${ }^{\mathrm{HA}}$-only transfectants. Blots of whole-cell and immunodepleted lysates showed patterns of protein expression and depletion consistent with specific Myc immunoprecipitation (Fig 1b). Lack of detectable $\mathrm{Csk}^{\mathrm{HA}}$ depletion from lysates with anti-Myc (i.e., substoichiometric binding of Csk ${ }^{\mathrm{HA}}$ to $\mathrm{Csk}{ }^{\mathrm{Myc}}$ ) is consistent with the moderately low affinity and fast on/off kinetics of dimerization in solution ${ }^{43}$, potential competition with endogenous PTPN22, and signal interference from $\mathrm{Csk}^{\mathrm{Myc}} / \mathrm{Csk}^{\mathrm{Myc}}$ and $\mathrm{Csk}^{\mathrm{HA}} / \mathrm{Csk}^{\mathrm{HA}}$ dimers.

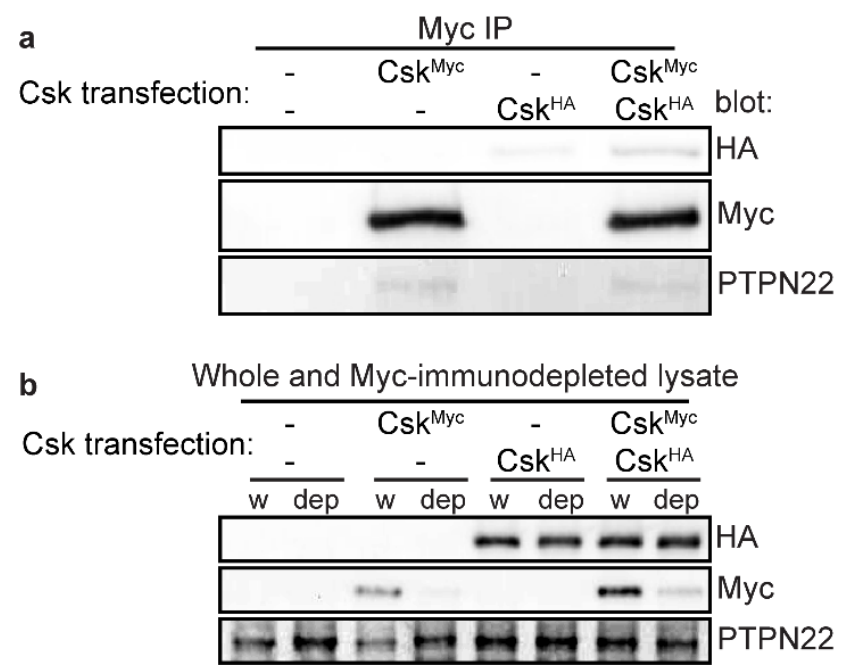

Fig. 1. Csk self-associates when overexpressed in Jurkat cells. (a) Immunoblots of anti-Myc immunoprecipitates (IP) from transiently transfected Jurkat T cells. Transfection conditions are indicated, including epitope-tagged Csk constructs (Csk ${ }^{\text {Myc }}$, $\mathrm{Csk}^{\mathrm{HA}}$ ) and empty pEF6 vector (-). Co-immunoprecipitation of endogenous PTPN22 is also shown. All data represent at least three independent experiments. (b) Immunoblots showing transfected Csk ${ }^{\text {Myc }}$ and $\mathrm{Csk}^{\mathrm{HA}}$ and endogenous PTPN22 in Jurkat whole-cell lysate (w) and Myc-immunodepleted lysate (dep) corresponding to the immunoprecipitation samples above. 
An amino-acid substitution in the Csk SH3 domain impairs homodimerization and enhances PTPN22 binding The SH3 domain of Csk contains Csk- and PTPN22-binding surfaces ${ }^{13,43,52}$ (Fig. 2a). Although the footprints of these interfaces overlap substantially, we identified unique interactions via structural alignment of the extended PTPN22 binding surface from published crystal and NMR structures ${ }^{13,44,52}$. A single residue, $\mathrm{H} 21$, was situated in the symmetrical homodimer interface but not in the PTPN22 interface (Fig. 2b). Analysis with Pymol software suggested that most amino acids could rearrange to non-clashing rotamers if swapped into this position; $\beta$-branched isoleucine was an exception. We therefore generated $\mathrm{H} 21 \mathrm{I}$ variants of $\mathrm{Csk}^{\mathrm{Myc}}$ and $\mathrm{Csk}{ }^{\mathrm{HA}}$ and tested their effect on Csk co-immunoprecipitation.

a

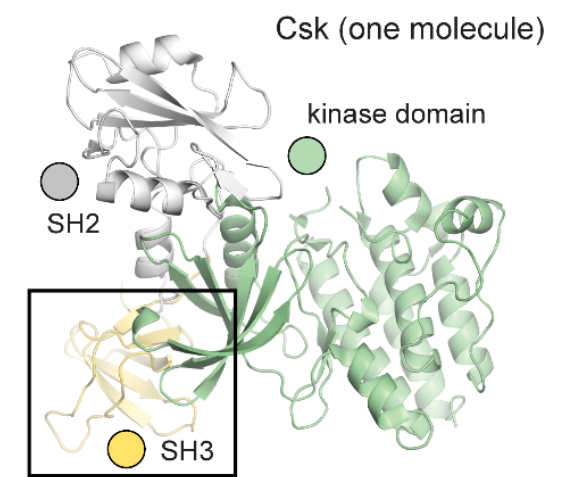

b

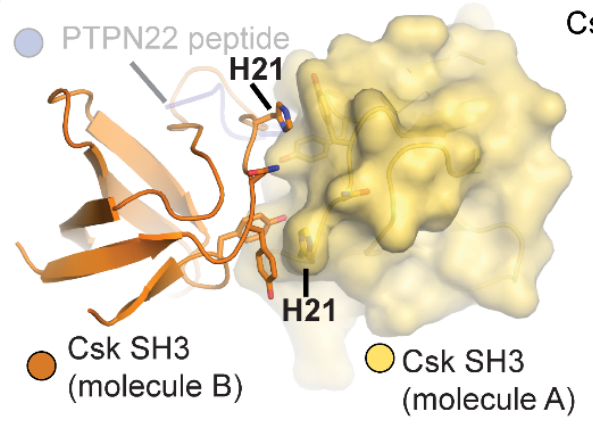

C Whole and Myc-immunodepleted lysate

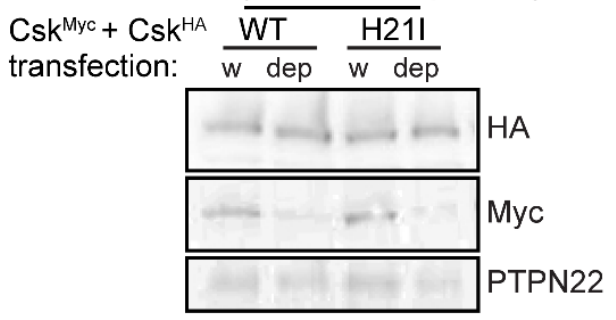

d
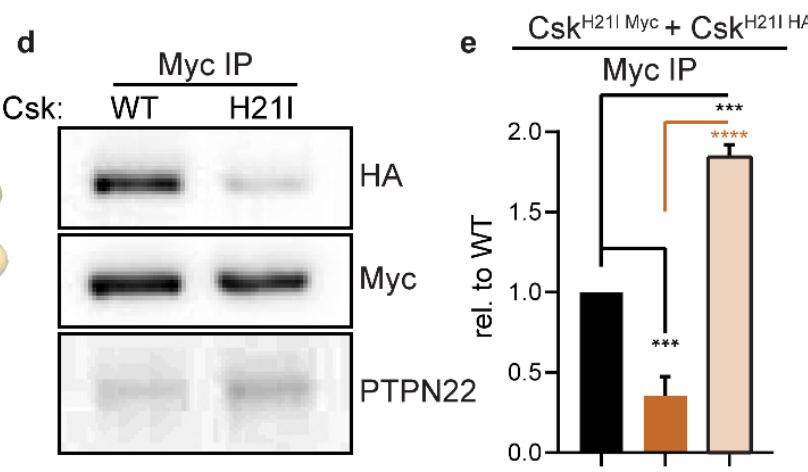

Blot: Myc HA PTPN22

Fig 2. H21I substitution in the SH3 domain of Csk selectively disrupt self-association. (a) Position of the SH3 domain in full-length Csk (protein databank identifier (PDB) $1 \mathrm{~K}^{4} \mathrm{~A}^{41}$ ). (b) Csk residue $\mathrm{H} 21$ in the SH3/SH3 homodimer interface (PDB $1 \mathrm{CSK}^{47}$ ). The position of PTPN22 peptide binding to the SH3 domain was derived in Pymol using PDBs $1 \mathrm{~K}^{2} \mathrm{~A}^{51} 1 \mathrm{CSK}^{47}$, 1JEG ${ }^{14}, 1 \mathrm{QWE} \mathrm{E}^{45}$, and $2 \mathrm{P} \mathrm{X}^{60}$. (c) Representative immunoblots of whole-cell or Myc-immunodepleted lysates from Jurkat cells transfected with Csk ${ }^{\mathrm{HA}}$ and Csk ${ }^{*} \mathrm{Myc}$ constructs ("both WT or both H21I). (d) Corresponding immunoblots of transfected Csk and endogenous PTPN22 in Myc immunoprecipitates. (e) Quantifications from immunoprecipitate blots, corrected for Csk ${ }^{\text {Myc }}$ pulldown in the same sample, shown relative to WT. Error bars: standard error of the mean (SEM), $n=4$. Significance: one-way ANOVA with Tukey's multiple comparison test (Sig.ANOVA) ${ }^{* * * \star} p<0.0001,{ }^{* * *} p=0.0001$ or 0.0008 .

We transiently transfected Jurkat cells with pairs of Csk ${ }^{\mathrm{Myc}}$ and Csk ${ }^{\mathrm{HA}}$ constructs, both either WT or H21I. Protein expression was not altered by $\mathrm{H} 21$ I substitution, and the Myc-tagged construct was effectively immunodepleted, as in WT (Fig. 2c). H21l substitution disrupted Csk ${ }^{\mathrm{HA}}$ co-immunoprecipitation with Csk ${ }^{\mathrm{Myc}}$ (60 $\pm 20 \%$ less HA-tagged H21I than WT) (Fig. 2d-e). This deficit in was accompanied by increased coimmunoprecipitation of endogenous PTPN22 (80 $\pm 20 \%$ more in H21-transfected cells than in WT-transfected cells). Together, these data suggest that Csk dimerization can compete with PTPN22 binding. This competition also suggests that the extended SH3-binding motif in the PTPN22 peptide, absent in PTPN12 ${ }^{10}$, cannot bind stably to the $\mathrm{SH} 3$ domain of Csk when homodimer formation blocks the canonical PXXP binding site. 
Although a previous study probed the $\mathrm{SH} 3$ homodimer interface using point mutations ${ }^{43}$, the authors did not test the effect of $\mathrm{H} 21$ I substitution. We purified recombinant, Csk WT and $\mathrm{H} 21 \mathrm{I}$ from bacteria and assessed their mobility on a size exclusion column. As expected, purified Csk WT and H21I were each visible as a single SDS PAGE band of $\sim 50 \mathrm{kDa}$, consistent with the expected $50.6 \mathrm{kDa}$ molecular weight of full-length Csk (Fig. 3a inset). Csk H21l, however, was retained longer than WT on a size exclusion column (Fig 3a), reflecting a decrease in apparent molecular weight $44,53,54$. Csk H21I, at $\sim 50 \mathrm{kDa}$, moves through the column as a monomer (Fig 3b) $)^{43,53,54}$. The apparent molecular weight of WT Csk is smaller than that expected for a stable dimer ( 70 $\mathrm{kDa}$ rather than $101 \mathrm{kDa}$ ), reflecting the rapidly exchanging population of monomers and dimers after dilution in the column ${ }^{43}$. Together the co-immunoprecipitation of Csk ${ }^{\mathrm{HA}}$ with $\mathrm{Csk}^{\mathrm{Myc}}$ and the observation that a structureguided point mutation selectively disrupts this interaction, in cells and in purified, recombinant protein, lead us to conclude that Csk homodimerizes in Jurkat T cells.

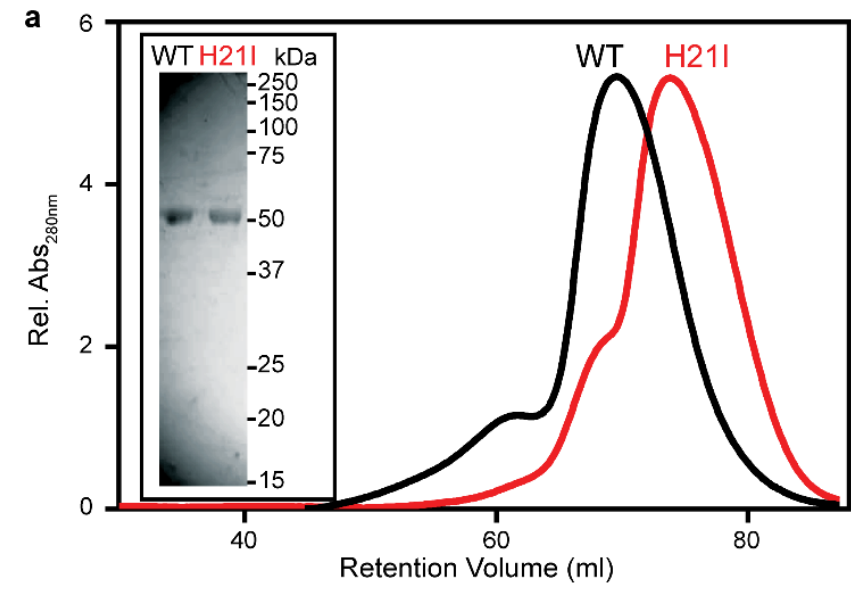

Fig. 3. Mutation of the homodimer interface decreases the apparent molecular weight of Csk in solution. (a) Colloidal blue staining (reducing SDS PAGE, inset) and Superdex 200 size-exclusion column migration of purified, recombinant Csk WT and H21I. (b) The apparent molecular weight of each Csk variant in solution was approximated based on the column partitioning of proteins with known molecular weights.

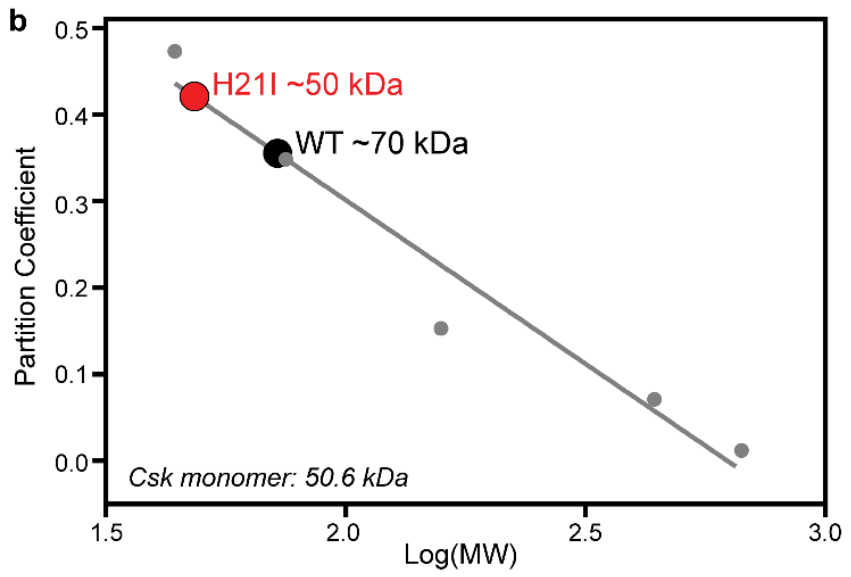

An amino-acid substitution in the Csk SH3 domain selectively impairs PTPN22 binding

The homodimer interface in the Csk SH3 domain overlaps completely with the canonical PXXP-binding site shared by PTPN12 and PTPN22. A second point of interaction with Csk, however, is unique to PTPN22, conserved in human LYP and mouse Pep ${ }^{43}$. Structural modeling of this extended interaction suggested that K43 in the Csk SH3 domain uniquely participates in PTPN22 binding but not homodimer formation (Fig. 4a). Since this positively charged residue forms a salt bridge with a nearby aspartate in PTPN22, we flipped the charge and minimized the degrees of rotameric freedom by mutation to an aspartate.

Csk K43D co-immunoprecipitated endogenous PTPN22 poorly $(70 \% \pm 30 \%$ less than WT), with no significant defect in $\mathrm{Csk}^{\mathrm{HA}}$ co-immunoprecipitation (Fig. 4b-c). The ability to selectively disrupt PTPN22-Csk coimmunoprecipitation further suggests that the Csk-Csk interaction is bimolecular and functions independently of a broader signaling complex. 
We also generated Myc- and HA-tagged constructs of Csk W47A, which has previously been used to ablate the binding of PXXP-containing ligands, including PTPN22, to the SH3 domain of Csk ${ }^{46-50}$. Residue W47 forms one of the proline binding pockets for the canonical PXXP interaction and interacts with the homodimerization footprint (Fig. 4a). Co-immunoprecipitation experiments confirmed that the Csk W47A variant had impairments in Csk/Csk and Csk/PTPN22 interactions (Fig. 4d-e).
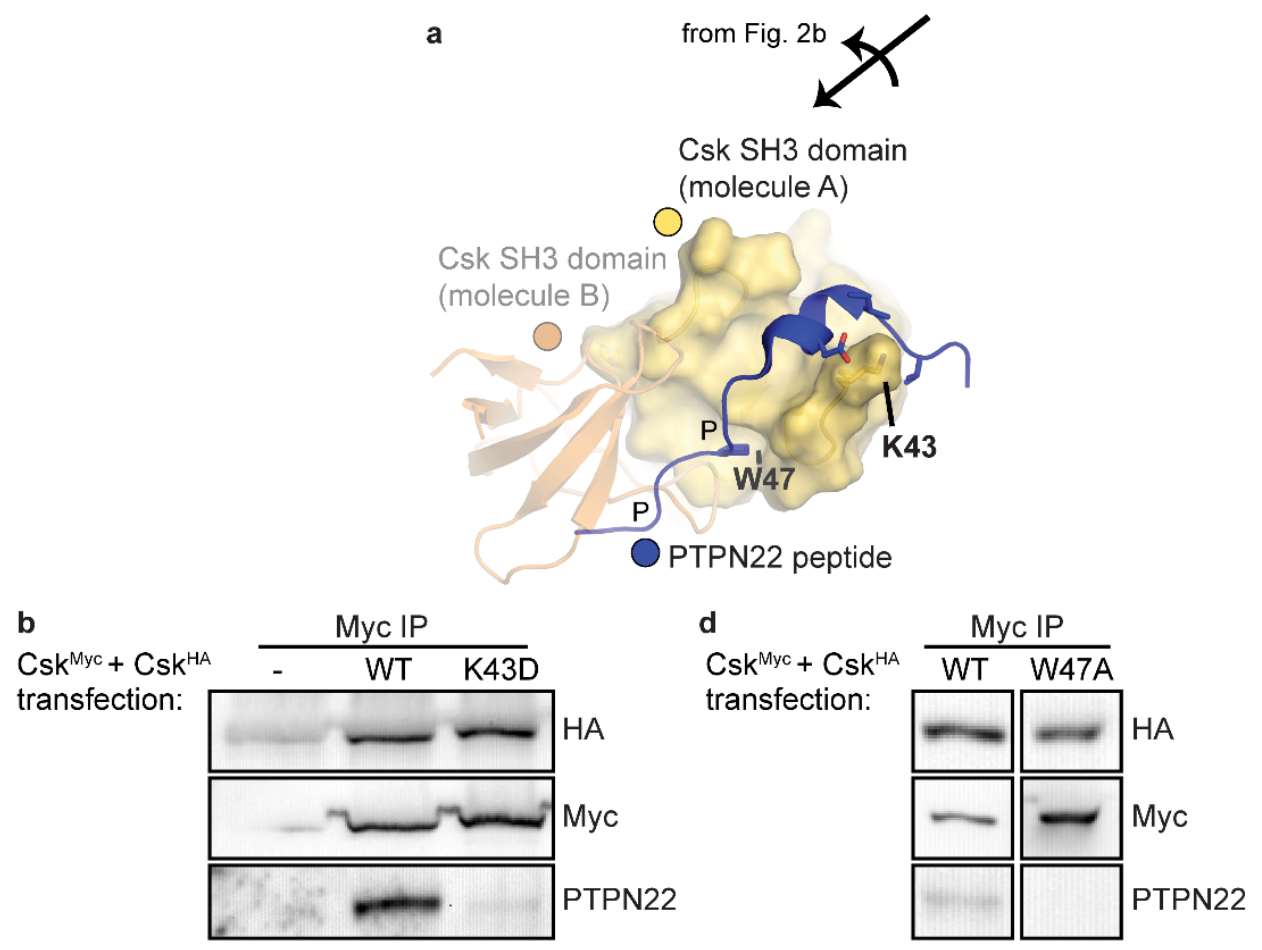

C

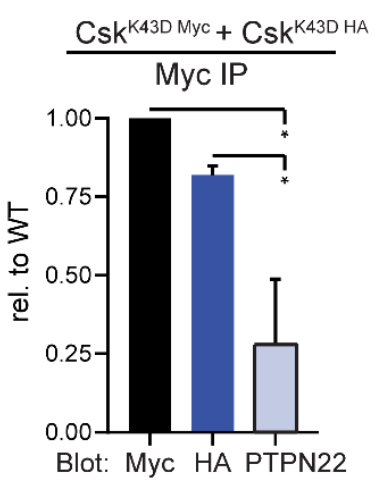

e

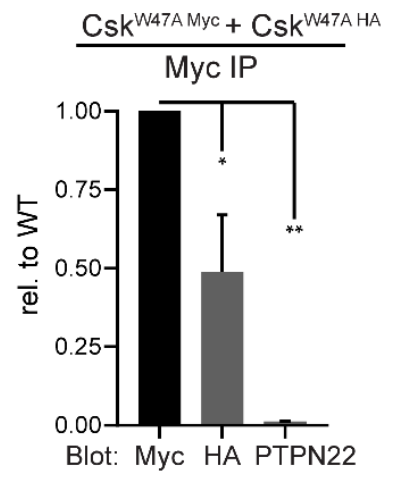

Fig. 4. K43D substitution in the SH3 domain of Csk selectively disrupts PTPN22 binding but not self-association, whereas W47A substitution impairs both interactions. (a) Csk residue K43D in the secondary binding interface between PTPN22 and the SH3 domain of Csk. The location of the proline $(\mathrm{P})$ residues within the peptide PXXP motif is shown, overlapping the dimer footprint; only one of the proline residues is engaged in this structure. Csk residue W47 is an essential component of the indicated proline binding pocket. (b) Representative immunoprecipitate blots from Jurkat cells transfected with Csk ${ }^{*}{ }^{H A}$ and Csk ${ }^{*}{ }^{M c}$ constructs ("both WT or both K43D). (c) Quantifications from immunoprecipitate blots, corrected for $\mathrm{Csk}^{\text {Myc }}$ pulldown in the same sample, shown relative to WT. Error bars: SEM, $n=3$. Sig.ANOVA * $p=0.0133$ or 0.0451 . (d) Representative immunoblots of immunoprecipitates from Jurkat cells transfected with Csk ${ }^{*}$ a and Csk ${ }^{*}$ Myc constructs ("both WT or both W47A). Boxed images were cropped from non-adjacent lanes of the same blot; image corrections for brightness and contrast were applied to the whole blot before cropping. (e) Quantifications from immunoprecipitate blots, corrected as above. Error bars: SEM, $n=4$. Sig.ANOVA ** $p=0.0010,{ }^{*} p=0.0282$.

H21I and K43D substitutions do not impair the kinase activity of Csk in solution

Purified WT, H21I, K43D, and kinase-impaired lysine-to-arginine mutant K222R ${ }^{3}$ Csk proteins were tested for activity against an optimal substrate peptide ${ }^{55}$ in a continuous spectrometric assay coupling ATP hydrolysis to $\mathrm{NADH}$ oxidation ${ }^{56}$. Kinase activity in solution, reflected in ADP production and decreasing absorbance at 340 
nm (Fig. 5a), was not impaired by either H21I or K43D substitution (Fig. 5b). As expected, Csk K222R was almost completely kinase dead $(94 \% \pm 5 \%$ loss of activity compared to WT).
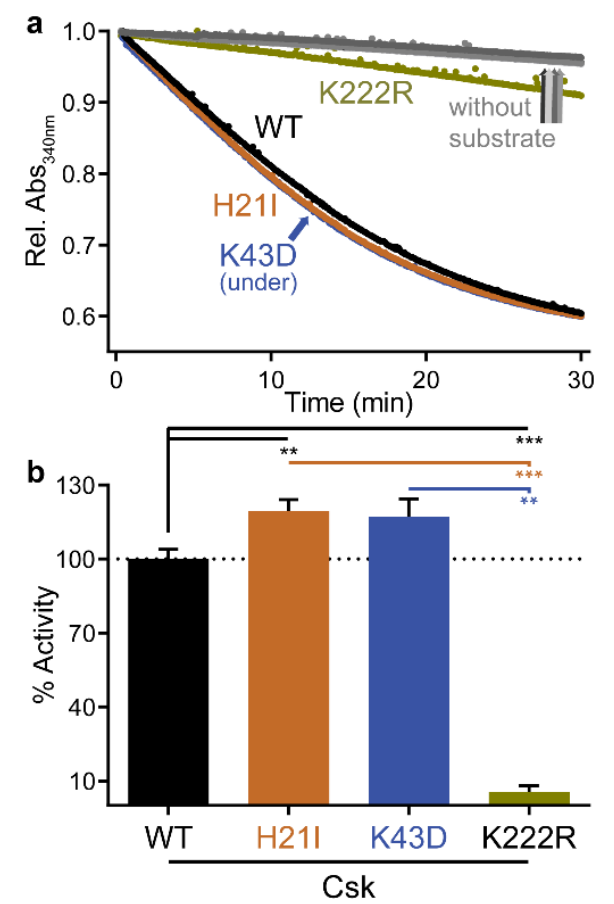

Fig. 5. H21I and K43D SH3-domain substitutions do not impair the catalytic activity of Csk in solution.

(a) Continuous spectrophotometric assay of peptide phosphorylation, in which ATP hydrolysis is coupled via pyruvate kinase and lactate dehydrogenase to NADH oxidation and loss of absorbance at $340 \mathrm{~nm}$. Reactions were initiated by adding Csk (WT, H211, $\mathrm{K} 43 \mathrm{D}$, or K222R). Control reactions in the absence of substrate peptide are also shown. (b) Linear velocities of the initial kinase reaction relative to WT. Error bars: SEM, $\mathrm{n}=4$.

\section{Discussion}

We present the first cellular evidence that Csk can form homodimers that compete with PTPN22 (LYP/Pep) binding. We used structural analysis to design single-amino-acid substitutions in the SH3 domain of Csk that disrupt either homodimer formation (Csk H21I) or PTPN22 binding (K43D) without impairing kinase activity. We also tested the substitution commonly used to block SH3-domain PXXP binding (Csk W47A) and found a secondary effect of impairing Csk homodimer formation. The more PTPN22-specific Csk K43D will therefore be a useful tool for selective studies of homodimer vs. phosphatase interactions in cell signaling. Together, the $\mathrm{H} 21 \mathrm{I}$ and K43D mutations demonstrate that PTPN22 binding and Csk homodimer formation compete for access to the Csk SH3 domain. This may indicate that multivalent recruitment of Csk to adaptors and other binding partners at the plasma membrane controls the residency of phosphatases alongside their membranelocalized substrates.

Future studies of Csk at physiological expression levels and in primary (non-cancer) cells will be necessary for quantitative analysis of homodimer formation. However, even at physiological expression levels Csk is likely at sufficiently high local concentration to drive homodimer formation ${ }^{57}$. CBP/PAG1 oligomerizes upon phosphorylation ${ }^{1,4-6}$ and, the signaling complexes in the early TCR signalosome are highly oligomeric and bridged. In our experiments, moreover, endogenously expressed proteins would have recruited and concentrated Csk at the membrane, limiting the effect of Csk overexpression on access to substrate.

The effect of homodimerization on Csk kinase activity remains an interesting question. We found no evidence that Csk H21I had impaired kinase activity, but the analysis was performed at a concentration below the likely $\mathrm{K}_{\mathrm{d}}$ of homodimer formation. Many tyrosine kinases have complex allosteric networks, including Csk, in which the $\mathrm{SH} 2$ domain activates the kinase when bound to phosphopeptide ${ }^{1,5,45}$. SH3-SH3 homodimer interactions could similarly influence Csk kinase activity.

We speculate that Csk homodimerization could be a key step in cell signaling. At equilibrium and relatively low concentration in the cytosol, the Csk SH3 domain could bind preferentially to phosphatase. In T cells, half of all PTPN22 molecules are associated with Csk, so this would be a substantial proportion of the available phosphatase ${ }^{19}$. Upon recruitment to the membrane, the local concentration of Csk would increase due to enrichment in microdomains and CBP/PAG1 oligomerization, and Csk homodimers could outcompete 
PTPN22. Released near its substrates, PTPN22 would then be free to dephosphorylate and block signaling through Lck, ITAMs, and Zap70. In this gain-of-function mode, the catalytically efficient phosphatase could act on many more substrate molecules after dissociation from the longer-lived Csk/Cbp/Lck complex. Alternatively, in a loss-of-function mode, PTPN22 might bind an alternative ligand (for instance, TRAF3, FAK or Pyk2 kinase $^{6}$, a Dok-family adaptor protein ${ }^{7}$ ) and lose access to substrates. It is also possible that a secondary effect of the autoimmunity risk allele PTPN22 R620W could be to increase Csk homodimer formation by loss of competition for SH3-domain access. Future studies will be required to address these questions.

Our structural observations also predict a functional difference between PTPN12 and PTPN22 phosphatases, possibly leading to differential regulation of SFK signaling in non-hematopoietic and hematopoietic cells. The PXXP-only PTPN12/Csk interaction, for instance, may be easily outcompeted by homodimer formation at a modest local concentration of Csk. PTPN12 binds to Csk only 1-10x more tightly than does another Csk molecule, and the PTPN12 binding site is completely occluded by the Csk/Csk interface, preventing rebinding. Hematopoietic-cell PTPN22, in contrast, may be more difficult to outcompete. PTPN22 binds to Csk 10-100x more tightly than does another molecule of Csk, and part of the PTPN22 binding site remains exposed even when Csk homodimer is formed. This could facilitate PTPN22 rebinding to Csk and require higher local concentrations of Csk for phosphatase release. These dynamics, in turn, could regulate the signaling thresholds or feedback regulation of the SFKs by cell type, by local concentrations of Csk-binding proteins at the plasma membrane, and by Csk expression itself.

In summary, we report that Csk can homodimerize in Jurkat T cells, in competition with the phosphatase PTPN22. We also present the Csk variants $\mathrm{H} 21 \mathrm{I}$ and K43D, new tools for uncoupling these binding processes. Csk expression levels, activity, and localization are all important regulators of signaling ${ }^{42,58-65}$ and can be disrupted in autoimmune disease ${ }^{25}$ and cancer ${ }^{22-24}$. The effect of these variables on homodimerization and PTPN22 binding will be interesting avenues for future mechanistic analysis.

\section{Materials and Methods}

Structural modeling

Structure-guided mutations were designed from models generated in PyMol (Schrödinger, New York, NY). Alignment and modeling of available NMR and crystal structures revealed amino acids that could be substituted to selectively disrupt homodimerization or PTPN22 binding. The homodimer interface was taken from a crystal structure of the isolated $\mathrm{SH} 3$ domain of Csk, in which crystal contacts are formed by the dimerization surface (PDB ID: 1CSK) ${ }^{52}$. The PTPN22-Csk interface was modeled as an amalgam of (i) an NMR structure of the SH3 domain from Csk bound to the 3BP1 peptide from PTPN22/Pep (PDB ID: 1JEG) ${ }^{13}$; and (ii) an NMR structure of the SH3 domain of Src kinase bound to the PXXP-containing peptide APP12 (PDB ID: 1QWE ${ }^{44}$, with a comparably oriented, canonical PXXP-SH3 interaction. Crystal structures of full-length Csk (PDB ID: 1K9A) ${ }^{66}$ and the phosphatase domain of PTPN22/Pep (PDB ID: 2P6X) ${ }^{67}$ were used to verify the placement of the binding surfaces. Figures were generated with Adobe software (San Jose, CA).

\section{DNA constructs}

Full-length cDNA encoding mouse Csk was subcloned into pEF6 vector (Life Technologies) with a C-terminal linker SAGGSAGG ${ }^{65}$ followed by a Myc (EQKLISEEDL) or HA (YPYDVPDYA) epitope tag. The variants H21I, $\mathrm{K} 43 \mathrm{D}, \mathrm{W} 47 \mathrm{~A}^{46}$, and K222R ${ }^{3}$ were created using QuikChange II site-directed mutagenesis (Agilent).

\section{Cell culture and transfection}

The Jurkat T-cell line E6-1 was maintained in RPMI with 10\% heat-inactivated fetal calf serum (FCS) with 2 $\mathrm{mM}$ glutamine, penicillin, and streptomycin at $0.15-1 \mathrm{M}$ cells $/ \mathrm{ml}$ in vented flasks in a $37^{\circ} \mathrm{C}$ cell incubator. For transfections, batches of $15 \mathrm{M}$ log-phase cells in antibiotic-free medium were collected, washed, and resuspended in $300 \mu \mathrm{l}$ medium with $10 \mu \mathrm{g}$ empty vector DNA and $20 \mu \mathrm{g}$ total Csk DNA (e.g. $10 \mu \mathrm{g} \mathrm{Csk}{ }^{\mathrm{Myc}}+10$ $\mu \mathrm{g} \mathrm{Csk}{ }^{\mathrm{HA}}$ ). After $15 \mathrm{~min}$ incubation at room temperature, cells were transferred to a $0.4 \mathrm{~cm}$ cuvet for a $10 \mathrm{~ms}$ pulse of $300 \mathrm{~V}$ on a BTX Square-Wave electroporator (Bio-Rad). Cells were rested $15 \mathrm{~min}$ at room temperature and then transferred to $10 \mathrm{ml}$ antibiotic-free medium and incubated overnight. Viable cells were quantified with a ViCell counter (Beckman) ${ }^{62,68}$. Jurkat cells from our lab (gift from A. Weiss at UCSF) have tested negative for mycoplasma and been verified by STR profiling and TCR expression.

$\underline{\text { Immunoprecipitation and blotting }}$ 
Lysates of transfected Jurkat cells were made by incubating at least $8 \mathrm{M}$ cells $15 \mathrm{~min}$ on ice in lysis buffer containing $1 \%$ lauryl maltoside, $150 \mathrm{mM} \mathrm{NaCl}, 0.02 \% \mathrm{NaN}_{3}, 0.4 \mathrm{mM}$ ethylenediaminetetraacetic acid (EDTA, Sigma), protease inhibitors (Sigma), and $10 \mathrm{mM}$ Tris, pH 7.5 (Sigma) and then centrifuging at $4^{\circ} \mathrm{C}$ for another $15 \mathrm{~min}$. Supernatants were mixed $2 \mathrm{~h}$ at $4^{\circ} \mathrm{C}$ with Protein-A-conjugated sepharose beads (GE Healthcare) and anti-Myc antibody (9B11, Cell Signaling Tech \#2276). The beads were then washed in lysis buffer and boiled in reducing SDS PAGE sample buffer. Proteins were resolved by SDS PAGE and transferred to an Immobilon $P$ polyvinylidene fluoride or Immobilon-FL membrane (EMD Millipore) $)^{61}$. Membranes were blocked with 3\% bovine serum albumin (Sigma), and then incubated with the primary antibodies goat anti-human PTPN22/Lyp (AF3428, R\&D Biosciences), anti-Myc Biotinylated antibody (71D10, Cell Signaling Tech \#3946), or anti-HA Biotinylated antibody (C29F4, Cell Signaling Tech \#5017). Secondary incubations included horseradishperoxidase-conjugated rabbit anti-goat IgG $(\mathrm{H}+\mathrm{L})$ (Southern Biotech 6164-05), Streptavidin-HRP (Cell Signaling Tech \#3999), or LI-COR IRDye secondary antibodies (NC9744100, NC9523609, 926-68072) as appropriate, and blots were developed with the addition of Western Lightning enhanced chemiluminescence reagent (PerkinEImer) and imaged on a Kodak Imagestation or LICOR Odyssey. Densitometry was performed using NIH ImageJ software, and statistical analysis was performed in GraphPad Prism.

\section{Protein purification}

Full-length mouse Csk (WT, H21I, K43D, and K222R³) were subcloned into a pGEX-6P-3 vector (GE Healthcare) containing an N-terminal glutathione S-transferase (GST) affinity tag. Each construct was transformed into BL21(DE3) cells (Agilent), and expression was induced with $0.2 \mathrm{mM}$ isopropyl- $\beta$-D-thiogalactoside (IPTG) at $18^{\circ} \mathrm{C}$ overnight. The bacterial pellet was resuspended in GST binding buffer, $\mathrm{pH} 7.4$ (phosphate-buffered saline with $5 \mathrm{mM}$ EDTA and $5 \mathrm{mM}$ dithiothreitol (DTT)) and lysed by freeze/thaw, lysozyme treatment, and sonication by a Branson 450 Sonifier. All purification steps were performed on ice or at $4^{\circ} \mathrm{C}$, and all columns and proteases were obtained from GE Healthcare. Clarified lysate was passed through a GST Gravitrap column, and, after washing, GST-Csk was eluted at $\mathrm{pH} 8.0$ with $10 \mathrm{mM}$ reduced glutathione, $25 \mathrm{mM}$ Tris, $50 \mathrm{mM} \mathrm{NaCl}$, and $1 \mathrm{mM}$ DTT. The GST tag was cleaved with PreScission Protease (GE Healthcare) overnight. After buffer exchange by concentration and dilution, Csk was further purified by HiTrap $\mathrm{Q}$ anion exchange chromatography at $\mathrm{pH} 8.0(50 \mathrm{mM}$ Tris, $50-1000 \mathrm{mM} \mathrm{NaCl}$, and $1 \mathrm{mM}$ DTT) followed by a Superdex 200 size exclusion column in $100 \mathrm{mM} \mathrm{NaCl}, 10 \%$ Glycerol, and $50 \mathrm{mM}$ Tris, pH 8.0. Purified Csk was flash frozen in liquid nitrogen and stored at $-70^{\circ} \mathrm{C}$. Homogeneity and molecular weight of purified proteins were verified by SDS PAGE with colloidal blue staining (Life Technologies) and mass spectrometry. The concentration of purified Csk was determined from its absorbance at $280 \mathrm{~nm}$ using a molar absorptivity of $73800 \mathrm{M}^{-1} \mathrm{~cm}^{-1}$ as calculated by the ExPASy ProtParam tool ${ }^{53}$.

\section{Size Exclusion Chromatography}

Size exclusion chromatography was performed by loading $1 \mathrm{ml}(20 \mathrm{mg}, 400 \mu \mathrm{M})$ purified Csk (WT or H21I) onto a HiLoad 16/60 Superdex 200 column. Retention volumes were estimated from fits to Gaussian curves (Absorbance=amplitude $\left.{ }^{*} \exp \left(-0.5^{*}((\text { volume }- \text { mean retention volume }) / \text { standard deviation })^{\wedge} 2\right)\right)$ and the fit mean retention volume was used to calculate partition coefficients from the function (retention volume - void volume) / (column volume - void volume). The elution of blue dextran marked the void volume, and elution of high-salt buffer (measured by conductance) marked the column volume. To relate the partition coefficient to an apparent molecular weight, partition coefficients of standard proteins from the high-molecular-weight gel filtration calibration kit (GE Healthcare \#28403842) were plotted against Log(MW) and fit to a linear function using GraphPad Prism software.

\section{Kinase Activity Assay}

The activity of purified Csk was measured using a continuous spectrometric assay: ATP hydrolysis is coupled via pyruvate kinase and lactate dehydrogenase to NADH oxidation, which results in a decrease in absorbance at $340 \mathrm{~nm}^{56,69}$. Kinase activity was measured at $30^{\circ} \mathrm{C}$ with a Molecular Devices Spectramax 340PC spectrophotometer in a $75 \mu \mathrm{l}$ reaction with final concentrations of $2.5 \mu \mathrm{M}$ Csk, $55.7 \mathrm{U} / \mathrm{ml}$ pyruvate kinase, 78 $\mathrm{U} / \mathrm{ml}$ lactate dehydrogenase, $0.6 \mathrm{mg} / \mathrm{ml} \mathrm{NADH}, 1 \mathrm{mM}$ phosphoenolpyruvate, $250 \mu \mathrm{M}$ ATP, $10 \mathrm{mM}$ Tris $\mathrm{pH} 7.5$, $1 \mathrm{mM} \mathrm{MgCl}_{2}$, and $1 \mathrm{mM}$ tris(2-carboxyethyl)phosphine (TCEP). The reaction was initiated by adding a final concentration of $250 \mu \mathrm{M}$ Csk optimal peptide substrate (KKKKEEIYFFF ${ }^{55}$ ) synthesized by Elim Biopharmaceuticals (Hayward, CA). Negligible background activity was observed if substrate or kinase were omitted from the reaction. Kinase activity was obtained by fitting the initial segment of the decay curve to a 
linear function. After fitting, the raw data were normalized so that the maximum value was 1 to facilitate visual comparison. Statistical analysis was performed in GraphPad Prism.

\section{References}

1 Okada, M. Regulation of the SRC family kinases by Csk. Int J Biol Sci 8, 1385-1397, doi:10.7150/ijbs.5141 (2012).

2 Brown, M. T. \& Cooper, J. A. Regulation, substrates and functions of src. Biochim Biophys Acta 1287, 121-149, doi:0304-419X(96)00003-0 [pii] (1996).

Chow, L. M., Fournel, M., Davidson, D. \& Veillette, A. Negative regulation of T-cell receptor signalling by tyrosine protein kinase p50csk. Nature 365, 156-160, doi:10.1038/365156a0 (1993).

Davidson, D., Bakinowski, M., Thomas, M. L., Horejsi, V. \& Veillette, A. Phosphorylation-dependent regulation of T-cell activation by PAG/Cbp, a lipid raft-associated transmembrane adaptor. Mol Cell Biol 23, 2017-2028, doi:10.1128/MCB.23.6.2017-2028.2003 (2003).

Kawabuchi, M. et al. Transmembrane phosphoprotein Cbp regulates the activities of Src-family tyrosine kinases. Nature 404, 999-1003, doi:10.1038/35010121 (2000).

6 Sabe, H., Hata, A., Okada, M., Nakagawa, H. \& Hanafusa, H. Analysis of the binding of the Src homology 2 domain of Csk to tyrosine-phosphorylated proteins in the suppression and mitotic activation of c-Src. Proc Natl Acad Sci U S A 91, 3984-3988 (1994).

Shah, K. \& Shokat, K. M. A chemical genetic screen for direct v-Src substrates reveals ordered assembly of a retrograde signaling pathway. Chem Bio/ 9, 35-47, doi:10.1016/s1074-5521(02)00086-8 (2002).

Levinson, N. M., Seeliger, M. A., Cole, P. A. \& Kuriyan, J. Structural basis for the recognition of c-Src by its inactivator Csk. Cell 134, 124-134, doi:10.1016/j.cell.2008.05.051 (2008).

Cloutier, J. F., Chow, L. M. \& Veillette, A. Requirement of the SH3 and SH2 domains for the inhibitory function of tyrosine protein kinase p50csk in T lymphocytes. Mol Cell Biol 15, 5937-5944 (1995).

Cloutier, J. F. \& Veillette, A. Association of inhibitory tyrosine protein kinase p50csk with protein tyrosine phosphatase PEP in T cells and other hemopoietic cells. EMBO J 15, 4909-4918 (1996).

11 Lin, X., Wang, Y., Ahmadibeni, Y., Parang, K. \& Sun, G. Structural basis for domain-domain communication in a protein tyrosine kinase, the C-terminal Src kinase. J Mol Biol 357, 1263-1273, doi:10.1016/j.jmb.2006.01.046 (2006).

12 Davidson, D., Cloutier, J. F., Gregorieff, A. \& Veillette, A. Inhibitory tyrosine protein kinase p50csk is associated with protein-tyrosine phosphatase PTP-PEST in hemopoietic and non-hemopoietic cells. J Biol Chem 272, 23455-23462 (1997).

13 Ghose, R., Shekhtman, A., Goger, M. J., Ji, H. \& Cowburn, D. A novel, specific interaction involving the Csk SH3 domain and its natural ligand. Nat Struct Biol 8, 998-1004, doi:10.1038/nsb1101-998 (2001).

14 Cohen, S., Dadi, H., Shaoul, E., Sharfe, N. \& Roifman, C. M. Cloning and characterization of a lymphoidspecific, inducible human protein tyrosine phosphatase, Lyp. Blood 93, 2013-2024 (1999).

15 Wang, H. et al. ZAP-70: an essential kinase in T-cell signaling. Cold Spring Harb Perspect Biol 2, a002279, doi:10.1101/cshperspect.a002279 (2010).

16 Straus, D. B. \& Weiss, A. Genetic evidence for the involvement of the lck tyrosine kinase in signal transduction through the T cell antigen receptor. Cell 70, 585-593, doi:0092-8674(92)90428-F [pii] (1992). 
17 Nika, K. et al. Constitutively active Lck kinase in T cells drives antigen receptor signal transduction. Immunity 32, 766-777, doi:10.1016/j.immuni.2010.05.011 (2010).

18 Wu, J. et al. Identification of substrates of human protein-tyrosine phosphatase PTPN22. J Biol Chem 281, 11002-11010, doi:10.1074/jbc.M600498200 (2006).

19 Cloutier, J. F. \& Veillette, A. Cooperative inhibition of T-cell antigen receptor signaling by a complex between a kinase and a phosphatase. J Exp Med 189, 111-121 (1999).

20 Gjorloff-Wingren, A., Saxena, M., Williams, S., Hammi, D. \& Mustelin, T. Characterization of TCR-induced receptor-proximal signaling events negatively regulated by the protein tyrosine phosphatase PEP. Eur J Immunol 29, 3845-3854, doi:10.1002/(SICl)1521-4141(199912)29:12\&\#60;3845::AIDIMMU3845\&\#62;3.0.CO;2-U (1999).

21 Ashouri, J. F., Lo, W. L., Nguyen, T. T. T., Shen, L. \& Weiss, A. ZAP70, too little, too much can lead to autoimmunity. Immunol Rev, doi:10.1111/imr.13058 (2021).

22 Sirvent, A., Benistant, C. \& Roche, S. Oncogenic signaling by tyrosine kinases of the SRC family in advanced colorectal cancer. Am J Cancer Res 2, 357-371 (2012).

23 Nakagawa, T. et al. Overexpression of the csk gene suppresses tumor metastasis in vivo. Int $J$ Cancer 88, 384-391 (2000).

24 Kanou, T. et al. The transmembrane adaptor Cbp/PAG1 controls the malignant potential of human nonsmall cell lung cancers that have c-src upregulation. Mol Cancer Res 9, 103-114, doi:10.1158/15417786.MCR-10-0340 (2011).

25 Manjarrez-Orduno, N. et al. CSK regulatory polymorphism is associated with systemic lupus erythematosus and influences B-cell signaling and activation. Nat Genet 44, 1227-1230, doi:10.1038/ng.2439 (2012).

26 Gregorieff, A., Cloutier, J. F. \& Veillette, A. Sequence requirements for association of protein-tyrosine phosphatase PEP with the Src homology 3 domain of inhibitory tyrosine protein kinase p50(csk). J Biol Chem 273, 13217-13222 (1998).

27 Begovich, A. B. et al. A missense single-nucleotide polymorphism in a gene encoding a protein tyrosine phosphatase (PTPN22) is associated with rheumatoid arthritis. Am J Hum Genet 75, 330-337, doi:10.1086/422827 (2004).

28 Totaro, M. C. et al. PTPN22 1858C>T polymorphism distribution in Europe and association with rheumatoid arthritis: case-control study and meta-analysis. PLoS One 6, e24292, doi:10.1371/journal.pone.0024292 (2011).

29 de Lima, S. C., Adelino, J. E., Crovella, S., de Azevedo Silva, J. \& Sandrin-Garcia, P. PTPN22 1858C > $\mathrm{T}$ polymorphism and susceptibility to systemic lupus erythematosus: a meta-analysis update. Autoimmunity 50, 428-434, doi:10.1080/08916934.2017.1385774 (2017).

30 Tizaoui, K. et al. Association of PTPN22 1858C/T Polymorphism with Autoimmune Diseases: A Systematic Review and Bayesian Approach. J Clin Med 8, doi:10.3390/jcm8030347 (2019).

31 Bottini, N. et al. A functional variant of lymphoid tyrosine phosphatase is associated with type I diabetes. Nat Genet 36, 337-338, doi:10.1038/ng1323 (2004).

32 Castro-Sanchez, P., Teagle, A. R., Prade, S. \& Zamoyska, R. Modulation of TCR Signaling by Tyrosine Phosphatases: From Autoimmunity to Immunotherapy. Front Cell Dev Biol 8, 608747, doi:10.3389/fcell.2020.608747 (2020). 
33 Vang, T. et al. LYP inhibits T-cell activation when dissociated from CSK. Nat Chem Biol 8, 437-446, doi:10.1038/nchembio.916 (2012).

34 Vang, T. et al. Autoimmune-associated lymphoid tyrosine phosphatase is a gain-of-function variant. Nat Genet 37, 1317-1319, doi:10.1038/ng1673 (2005).

35 Yang, S. et al. PTPN22 phosphorylation acts as a molecular rheostat for the inhibition of TCR signaling. Sci Signal 13, doi:10.1126/scisignal.aaw8130 (2020).

36 Cao, Y. et al. High basal activity of the PTPN22 gain-of-function variant blunts leukocyte responsiveness negatively affecting IL-10 production in ANCA vasculitis. PLoS One 7, e42783, doi:10.1371/journal.pone.0042783 (2012).

37 Perry, D. J. et al. Overexpression of the PTPN22 Autoimmune Risk Variant LYP-620W Fails to Restrain Human CD4(+) T Cell Activation. J Immunol 207, 849-859, doi:10.4049/jimmunol.2000708 (2021).

38 Hasegawa, K. et al. PEST domain-enriched tyrosine phosphatase (PEP) regulation of effector/memory T cells. Science 303, 685-689, doi:10.1126/science.1092138 (2004).

39 Clarke, F. et al. The protein tyrosine phosphatase PTPN22 negatively regulates presentation of immune complex derived antigens. Sci Rep 8, 12692, doi:10.1038/s41598-018-31179-x (2018).

40 Bottini, N. \& Peterson, E. J. Tyrosine phosphatase PTPN22: multifunctional regulator of immune signaling, development, and disease. Annu Rev Immunol 32, 83-119, doi:10.1146/annurev-immunol032713-120249 (2014).

41 Wang, Y. et al. The autoimmunity-associated gene PTPN22 potentiates toll-like receptor-driven, type 1 interferon-dependent immunity. Immunity 39, 111-122, doi:10.1016/j.immuni.2013.06.013 (2013).

42 Wallis, A. M. et al. TRAF3 enhances TCR signaling by regulating the inhibitors Csk and PTPN22. Sci Rep 7, 2081, doi:10.1038/s41598-017-02280-4 (2017).

43 Levinson, N. M., Visperas, P. R. \& Kuriyan, J. The tyrosine kinase Csk dimerizes through Its SH3 domain. PLoS One 4, e7683, doi:10.1371/journal.pone.0007683 (2009).

44 Feng, S., Kasahara, C., Rickles, R. J. \& Schreiber, S. L. Specific interactions outside the proline-rich core of two classes of Src homology 3 ligands. Proc Natl Acad Sci U S A 92, 12408-12415 (1995).

45 Takeuchi, S., Takayama, Y., Ogawa, A., Tamura, K. \& Okada, M. Transmembrane phosphoprotein Cbp positively regulates the activity of the carboxyl-terminal Src kinase, Csk. J Biol Chem 275, 29183-29186, doi:10.1074/jbc.C000326200 (2000).

46 Torgersen, K. M. et al. Release from tonic inhibition of T cell activation through transient displacement of C-terminal Src kinase (Csk) from lipid rafts. J Biol Chem 276, 29313-29318, doi:10.1074/jbc.C100014200 (2001).

47 de la Puerta, M. L. et al. The autoimmunity risk variant LYP-W620 cooperates with CSK in the regulation of TCR signaling. PLoS One 8, e54569, doi:10.1371/journal.pone.0054569 (2013).

48 Drobek, A. et al. PSTPIP2, a Protein Associated with Autoinflammatory Disease, Interacts with Inhibitory Enzymes SHIP1 and Csk. J Immunol 195, 3416-3426, doi:10.4049/jimmunol.1401494 (2015).

49 Vang, T., Abrahamsen, H., Myklebust, S., Horejsi, V. \& Tasken, K. Combined spatial and enzymatic regulation of Csk by cAMP and protein kinase a inhibits T cell receptor signaling. J Biol Chem 278, 1759717600, doi:10.1074/jbc.C300077200 (2003). 
50 Otahal, P., Pata, S., Angelisova, P., Horejsi, V. \& Brdicka, T. The effects of membrane compartmentalization of csk on TCR signaling. Biochim Biophys Acta 1813, 367-376, doi:10.1016/j.bbamcr.2010.12.003 (2011).

51 Pao, L. I., Badour, K., Siminovitch, K. A. \& Neel, B. G. Nonreceptor protein-tyrosine phosphatases in immune cell signaling. Annu Rev Immunol 25, 473-523, doi:10.1146/annurev.immunol.23.021704.115647 (2007).

52 Borchert, T. V., Mathieu, M., Zeelen, J. P., Courtneidge, S. A. \& Wierenga, R. K. The crystal structure of human CskSH3: structural diversity near the RT-Src and n-Src loop. FEBS Lett 341, 79-85, doi:00145793(94)80244-0 [pii] (1994).

53 Wilkins, M. R. et al. Protein identification and analysis tools in the ExPASy server. Methods Mol Biol 112, 531-552 (1999).

54 Klages, S. et al. Ctk: a protein-tyrosine kinase related to Csk that defines an enzyme family. Proc Natl Acad Sci U S A 91, 2597-2601 (1994).

55 Sondhi, D., Xu, W., Songyang, Z., Eck, M. J. \& Cole, P. A. Peptide and protein phosphorylation by protein tyrosine kinase Csk: insights into specificity and mechanism. Biochemistry 37, 165-172, doi:10.1021/bi9722960 (1998).

56 Barker, S. C. et al. Characterization of pp60c-src tyrosine kinase activities using a continuous assay: autoactivation of the enzyme is an intermolecular autophosphorylation process. Biochemistry 34, 1484314851 (1995).

57 Kholodenko, B. N. Cell-signalling dynamics in time and space. Nat Rev Mol Cell Biol 7, 165-176, doi:10.1038/nrm1838 (2006).

58 Wallis, A. M. \& Bishop, G. A. TRAF3 regulation of inhibitory signaling pathways in B and T lymphocytes by kinase and phosphatase localization. J Leukoc Biol, doi:10.1002/JLB.2MIR0817-339RR (2018).

59 Liang, F. et al. Translational control of C-terminal Src kinase (Csk) expression by PRL3 phosphatase. J Biol Chem 283, 10339-10346, doi:10.1074/jbc.M708285200 (2008).

60 Brian, B. F. \& Freedman, T. S. The Src-family Kinase Lyn in Immunoreceptor Signaling. Endocrinology 162, bqab152, doi:10.1210/endocr/bqab152 (2021).

61 Brian, B. F., Guerrero, C. R. \& Freedman, T. S. Immunopharmacology and Quantitative Analysis of Tyrosine Kinase Signaling. Current protocols in immunology / edited by John E. Coligan ... [et al.] 130, e104, doi:10.1002/cpim.104 (2020).

62 Brian, B. F. et al. Unique-region phosphorylation targets LynA for rapid degradation, tuning its expression and signaling in myeloid cells. eLife 8, e46043, doi:10.7554/eLife.46043 (2019).

63 Freedman, T. S. et al. LynA regulates an inflammation-sensitive signaling checkpoint in macrophages. eLife 4, e09183, doi:10.7554/eLife.09183 (2015).

64 Tan, Y. X. et al. Inhibition of the kinase Csk in thymocytes reveals a requirement for actin remodeling in the initiation of full TCR signaling. Nat Immunol 15, 186-194, doi:10.1038/ni.2772 (2014).

65 Schoenborn, J. R., Tan, Y. X., Zhang, C., Shokat, K. M. \& Weiss, A. Feedback circuits monitor and adjust basal Lck-dependent events in $\mathrm{T}$ cell receptor signaling. Sci Signal 4, ra59, doi:10.1126/scisignal.2001893 (2011).

66 Ogawa, A. et al. Structure of the carboxyl-terminal Src kinase, Csk. J Biol Chem 277, 14351-14354, doi:10.1074/jbc.C200086200 (2002). 
67 Barr, A. J. et al. Large-scale structural analysis of the classical human protein tyrosine phosphatome. Cell 136, 352-363, doi:10.1016/j.cell.2008.11.038 (2009).

68 Brian, B. F. et al. Splice-specific lyn knockout mice reveal a dominant function of LynB in preventing autoimmunity. bioRxiv, doi:10.1101/2021.05.03.439514 (2021).

69 Seeliger, M. A. et al. High yield bacterial expression of active c-Abl and c-Src tyrosine kinases. Protein Sci 14, 3135-3139, doi:10.1110/ps.051750905 (2005).

\section{Acknowledgements}

We thank Haichuan Liu and the UCSF Helen Diller Family Comprehensive Cancer Center Mass Spectrometry Core Facility for technical and analytical expertise. We thank Bianca Lee, Art Weiss, Erik Peterson, and

Nicholas Levinson for valuable discussions.

\section{Funding}

National Institutes of Health grant T32AR007304 (TSF)

National Institutes of Health grant R01AR073966 (TSF)

National Institutes of Health grant T32DA007097 (BFB)

University of Minnesota Foundation Equipment Award E-0918-01 (TSF)

\section{Author contributions}

Conceptualization: TSF

Methodology: TSF

Investigation: BFB, FVS, TSF

Visualization: BFB, TSF

Funding acquisition: BFB, TSF

Project administration: TSF

Supervision: TSF

Writing - original draft: BFB, FVS, TSF

Writing - review \& editing: BFB, FVS, TSF

\section{Additional Information}

Competing interests: The authors declare that they have no competing interests.

Data and materials availability: All data are available in the main text or the supplementary materials.

\section{Figure legends}

Fig. 1. Csk self-associates when overexpressed in Jurkat cells. (a) Immunoblots of anti-Myc immunoprecipitates (IP) from transiently transfected Jurkat T cells. Transfection conditions are indicated, including epitope-tagged Csk constructs ( $\mathrm{Csk}^{\mathrm{Myc}}, \mathrm{Csk}^{\mathrm{HA}}$ ) and empty pEF6 vector (-). Co-immunoprecipitation of endogenous PTPN22 is also shown. All data represent at least three independent experiments. (b) Immunoblots showing transfected Csk ${ }^{\mathrm{Myc}}$ and $\mathrm{Csk}^{\mathrm{HA}}$ and endogenous PTPN22 in Jurkat whole-cell lysate (w) and Myc-immunodepleted lysate (dep) corresponding to the immunoprecipitation samples above.

Fig 2. H21I substitution in the SH3 domain of Csk selectively disrupt self-association. (a) Position of the $\mathrm{SH} 3$ domain in full-length Csk (protein databank identifier (PDB) $1 \mathrm{~K}^{6}{ }^{66}$ ). (b) Csk residue $\mathrm{H} 21$ in the $\mathrm{SH} / \mathrm{SH} 3$ homodimer interface (PDB 1CSK ${ }^{52}$ ). The position of PTPN22 peptide binding to the $\mathrm{SH} 3$ domain was derived in Pymol using PDBs $1 \mathrm{~K}^{5} \mathrm{~A}^{56} 1 \mathrm{CSK}^{52}, 1 \mathrm{JEG}^{13}, 1 \mathrm{QWE}^{44}$, and $2 \mathrm{P} 6 \mathrm{X}^{67}$. (c) Representative immunoblots of wholecell or Myc-immunodepleted lysates from Jurkat cells transfected with Csk ${ }^{*} \mathrm{HA}$ and Csk ${ }^{*} \mathrm{Myc}$ constructs ("both WT or both $\mathrm{H} 21 \mathrm{I})$. (d) Corresponding immunoblots of transfected Csk and endogenous PTPN22 in Myc 
immunoprecipitates. (e) Quantifications from immunoprecipitate blots, corrected for Csk ${ }^{\text {Myc }}$ pulldown in the same sample, shown relative to WT. Error bars: standard error of the mean (SEM), $n=4$. Significance: one-way ANOVA with Tukey's multiple comparison test (Sig.ANOVA) ${ }^{* * * *} p<0.0001,{ }^{* * *} p=0.0001$ or 0.0008.

Fig. 3. Mutation of the homodimer interface decreases the apparent molecular weight of Csk in solution. (a) Colloidal blue staining (reducing SDS PAGE, inset) and Superdex 200 size-exclusion column migration of purified, recombinant Csk WT and H21I. (b) The apparent molecular weight of each Csk variant in solution was approximated based on the column partitioning of proteins with known molecular weights.

Fig. 4. K43D substitution in the SH3 domain of Csk selectively disrupts PTPN22 binding but not selfassociation, whereas W47A substitution impairs both interactions. (a) Csk residue K43D in the secondary binding interface between PTPN22 and the SH3 domain of Csk. The location of the proline $(\mathrm{P})$ residues within the peptide PXXP motif is shown, overlapping the dimer footprint; only one of the proline residues is engaged in this structure. Csk residue W47 is an essential component of the indicated proline binding pocket. (b) Representative immunoprecipitate blots from Jurkat cells transfected with Csk ${ }^{*}{ }^{A}$ and Csk $^{*}{ }^{* 1}$ constructs ("both WT or both K43D). (c) Quantifications from immunoprecipitate blots, corrected for Csk ${ }^{\text {Myc }}$ pulldown in the same sample, shown relative to WT. Error bars: SEM, $n=3$. Sig.ANOVA * $p=0.0133$ or 0.0451 . (d) Representative immunoblots of immunoprecipitates from Jurkat cells transfected with Csk ${ }^{*}{ }^{A}$ and Csk ${ }^{*}{ }^{M c}$ constructs ("both WT or both W47A). Boxed images were cropped from non-adjacent lanes of the same blot; image corrections for brightness and contrast were applied to the whole blot before cropping. (e) Quantifications from immunoprecipitate blots, corrected as above. Error bars: SEM, $n=4$. Sig. ANOVA ${ }^{* *} p=0.0010,{ }^{*} p=0.0282$.

Fig. 5. H21I and K43D SH3-domain substitutions do not impair the catalytic activity of Csk in solution. (a) Continuous spectrophotometric assay of peptide phosphorylation, in which ATP hydrolysis is coupled via pyruvate kinase and lactate dehydrogenase to NADH oxidation and loss of absorbance at $340 \mathrm{~nm}$. Reactions were initiated by adding Csk (WT, H21I, K43D, or K222R). Control reactions in the absence of substrate peptide are also shown. (b) Linear velocities of the initial kinase reaction relative to WT. Error bars: SEM, $n=4$. 